\title{
DESARROLLO DE UN BANCO DE DATOS SOBRE CARACTERISTICAS DE LOS ALIMENTOS ESPAÑOLES
}

\author{
Maria Teresa Fernández Muñoz", Carmen Galbán Ferrús*, Aida Méndez**, Luz \\ Moreno", Magdalena Ribas"*"
}

Resumen: Se describe el banco de datos sobre componentes de alimentos españoles: productos lácteos y vinos. Se ha desarrollado, en fase experimental, en el Instituto de Información y Documentación en Ciencia y Tecnología (ICYT), en colaboración con el Instituto de Fermentaciones Industriales, pertenecientes al CSIC. Además de información factual sobre componentes de alimentos, proporciona información adicional de tipo bibliográfico como: trabajos, autores, instituciones, métodos de análisis, etc., siempre en relación con los alimentos o sus componentes. La aplicación informática, desarrollada en DBIII, permite, mediante menús, la actualización, modificación y consulta de la información contenida en el banco de datos. Se comenta la normalización, organización y recuperación de la información.

Palabras clave: Banco de datos, componentes de alimentos, productos lácteos, vinos.

\begin{abstract}
The Spanish food composition Data Bank on dairy products and alcoholic beverages is described. A prototipe has been developed by the Institute for Information and Documentation in Science and Technology in collaboration with the Industrial Fermentation Institute, both of them belonging to the CSIC. Factual information as well as bibliografic information on authors, papers, institutions, analytical methods, etc., related in every case to food, are available through the data bank. The computer application using the DBIII, allows, by means of a menu system, the update and retrieval of the information on the data bank. Explanations on the standardization, organization and retrieval of information are included.
\end{abstract}

Keywords: Data bank, food composition, dairy products, alcoholic beverages.

\section{Introducción}

La información sobre la composición químico-física de los alimentos tiene influencia en muy diversos campos tanto a nivel nacional como internacional. Muchas y variadas actividades de tipo económico, educativo y social, desde el marketing a la investigación básica, necesitan basarse en el conocimiento profundo de la composición de los alimentos para la realización de su cometido. Es, por tanto, de gran importancia el poder contar con datos homologados que puedan servir de valores de referencia.

El primer colectivo que se interesó por los datos de composición de alimentos fue el sanitario, preocupado por la influencia de las dietas en la salud y en la enfermedad, como manifestaron en 1940 McCance y Widdowson (1).

* Instituto de Información y Documentación en Ciencia y Tecnologia, CSIC.

** Instituto de Estudios Avanzados CSIC-Universidad de las Islas Baleares.

*** Instituto de Fermentaciones Industriales, CSIC.

Recibido: $1-5-90$. 
Otros sectores de actividad demostraron su interés mucho más recientemente. A ello ha contribuido, entre otros factores, el aumento de la población mundial, las frecuentes inmigraciones, la escasez de alimentos, la mejora de la calidad de vida y el continuo incremento de las transacciones internacionales. En nuestro pais, la pertenencia a la CEE y la próxima integración total en 1993 supone un reto a la competitividad de nuestros productos, por lo que habrá que aumentar los controles que garanticen la homogeneidad y regularidad de su calidad y permitan la comparecencia en mercados cada vez más concurrentes y exigentes.

En la mayoría de los países industrializados se han creado tablas de composición de alimentos (2), que han venido recogiendo los valores analíticos de los componentes de los alimentos más usuales o con alguna significación para el pais productor. El Instituto de Nutrición del CSIC ha creado las tablas españolas que recogen valores de algunos componentes de 231 alimentos (3). Las tablas de composición de alimentos han sido, hasta hace poco, el único medio para satisfacer las necesidades de este tipo de información. Aunque muy útiles, son limitadas en cuanto a la cantidad y variabilidad de datos que pueden contener, así como a la actualidad de los mismos, dadas las dificultades que entraña su actualización.

El desarrollo de las tecnologías de la informática y telecomunicaciones ha posibilitado la creación de bases de datos de los componentes de los alimentos (47), muchas veces derivadas de las primitivas tablas ya existentes, y ha animado a países que carecían de ellas a emprender la tarea de su creación. Estos sistemas, capaces de almacenar y gestionar grandes volúmenes de información, permiten, además de un acceso y utilización fácil de los datos, reflejar más ágilmente sus variaciones, derivadas de la mejora de los métodos de análisis, de la evolución de las tecnologías de alimentos, así como de los cambios en las costumbres alimenticias.

Las facilidades informáticas y telemáticas han incrementado la utilización, intercambio y comparación entre los datos, tanto a nivel nacional como internacional, y han posibilitado el establecimiento de redes de bases de datos. Esto conlleva, además de un presupuesto considerable, una homogeneización de criterios en cuanto a técnicas de análisis y muestreo, expresión de resultados, identificación de los alimentos y componentes, etc., dadas las grandes diferencias que existen entre las distintas metodologias utilizadas por los distintos laboratorios y productores de datos.

Han surgido ya proyectos como FOODBASE (8) del U.S. National Cancer Institute que, conjuntamente con Technical Assessment Systems, Inc., están desarrollando una base de datos para recoger valorres sobre componentes y consumo de alimentos a nivel internacional; y otros proyectos tales como INFOODS (International Network of Food Data System) (9-11) y sus derivados como proyectos regionales EUROFOODS, NORFOODS o LATINFOODS (12), todos ellos apoyados por la UNU (Universidad de las Naciones Unidas). Tienen como objetivos principales promover la cooperación internacional para la adquisición, distribución y creación de un sistema internacional de datos de composición de alimentos, estableciendo criterios para la armonización general de la información sobre composición de alimentos. En Europa, bajo el programa FLAIR (Foods Linked Agro-Industrial Research), se han unido EUROFOODS y ENFANT (European Network on Food and Nutrition Tables) en el proyecto "Improvement 
of the quality and Compatibility of Food Consumption and Food Composition Data in Europe» (13). Este proyecto pretende establecer en la CEE una red de bases de datos de composición y consumo de alimentos nacionales comparables y compatibles, que facilite la obtención e intercambio de información.

En nuestro país no conocemos ningún banco de datos que recoja la información sobre composición de los alimentos españoles, aunque existen algunos específicos como los de vinos de Navarra, semillas del INIA, etc.

El desarrollo a nivel nacional de un banco de datos de este tipo que recoja los alimentos más usuales y característicos de España requiere la cooperación, tanto a nivel institucional de organismos e industrias, como a nivel personal, de investigadores y profesionales del sector. Con ello se podrían resolver los problemas que puedan plantearse y conseguir una armonización de criterios para el desarrollo de un sistema fiable, consistente y compatible con los existentes en nuestro entorno, pudiéndose derivar, además, tablas de composición a nivel nacional, regional o sectorial que satisfagan las necesidades de información de los científicos, industriales y profesionales del sector de la alimentación.

Ante la falta de una sistematizada recopilación de datos que definan y caractericen a los alimentos españoles, un grupo de investigadores del CSIC, pertenecientes a los Institutos de Fermentaciones Industriales y de Información y Documentación en Ciencia y Tecnología (ICYT), emprendieron la tarea, a través de un proyecto financiado por la CICYT, de la creación, en fase experimental, de un banco de datos que recogiese este tipo de información (14).

En este artículo se presenta la estructura y los módulos implementados de la base de datos desarrollada en el ICYT.

\section{Usuarios del Banco de Datos}

Como primera premisa para crear el banco de datos se hizo un estudio de sus usuarios potenciales. Según sus necesidades de información se clasificaron, a priori, en tres grupos:

- Investigadores de organismos de investigación, educación e industria. Estarán interesados principalmente en la determinación de los valores analíticos de los componentes de los alimentos, debidamente detallados y documentados en cuanto a métodos analíticos y de muestreo, estacionalidades, variedades de materia prima, condiciones de producción, almacenamiento, etc.

-. Profesionales de medicina, nutrición y dietética. Desearán conocer los valores analíticos medios de grupos determinados de componentes de alimentos específicos, usualmente utilizados para las dietas, ya sean de enfermos o de grupos de población.

- El tercer grupo de usuarios lo constituyen industriales de producción. de transformación y de distribución, economistas, administradores, legisladores, etc., cuyo interés principal estará localizado en el conocimiento de los valores medios de los componentes más característicos de los alimentos, complementados con información relativa a otras características de los mismos, tales como: zonas geográficas, origen, legislación, tratamientos, etc. 


\section{Contenido del Banco de Datos}

Son muchos los aspectos de los datos que deben tenerse en cuenta en la creación de un banco de datos, si se quiere satisfacer a un amplio espectro de usuarios. Aunque la información solicitada difiera, lo que sí necesitará cualquier usuario son datos precisos y fiables. Como la información se recoge de publicaciones científicas, informes de trabajos hechos en laboratorios públicos o privados, con distintas denominaciones de los alimentos y componentes, distintas metodologías de análisis y tratamiento de las muestras, distintas formas de expresar los resultados, etc., es necesaria su integración y homogeneización para obtener una información coherente. Para ello se deberá contar con un grupo de personas altamente cualificadas responsables de contrastar y normalizar la información que entra en el sistema.

La información contenida en el Banco de Datos se agrupa según los siguientes apartados.

1. Identificación de los alimentos.

2. Identificación de los componentes, su clasificación y medidas.

3. Identificación de las fuentes de información.

Estos tres grupos de información son dependientes unos de otros y relacionados entre sí.

La toma de datos comienza precisando la fuente, que será el documento de donde se extraen los datos. Esta puede ser artículo de revista, tesis doctoral, memorias, informes, libros, etc. Ello garantiza la identificación y el origen de los datos suministrados. Una vez detallada la fuente y aceptada por el sistema, después de un proceso de verificación, se precisa el alimento del que se quieren introducir datos, con los cualificadores que se crean oportunos. Tanto el nombre del alimento como los de los cualificadores deberán estar contenidos previamente en los ficheros maestros que constituyen el diccionario del sistema. Si no es así, se rechaza. Una vez aceptada la denominación del alimento, se expresarán debidamente los componentes y los valores analíticos de éstos. Resumiendo, un valor analítico estará unido a un componente de un alimento determinado y éste a una fuente concreta.

En todas las fases del funcionamiento del banco de datos y siempre que ha sido posible se ha comprobado la información introducida mediante ficheros testigos que garantizan el control del vocabulario.

Teniendo en cuenta que la iniciativa de la creación del Banco de Datos surgió dentro del proyecto coordinado «Caracterización de los Alimentos», se contó desde el principio con la colaboración de sus investigadores, por lo que el contenido y las posibles consultas a que diera lugar se establecieron con ellos.

Se decidió empezar, de forma experimental, por la creación de un banco de datos sobre productos lácteos y vinos.

\subsection{Identificación de los alimentos}

Una de las mayores dificultades en la creación y uso de un banco de datos de alimentos proviene de la inconsistencia de las denominaciones y descripciones de 
los elementos en ella considerados, ya que un nombre de un alimento puede tener distintas significaciones dependiendo del contexto geográfico, cultural y social. Por tanto, es primordial el establecer criterios y guias que garanticen una correcta e inequívoca identificación de los alimentos, sus constituyentes, metodologias de análisis, etc., que permitan compatibilizar la información que, proviniendo de distintas fuentes, va a incorporarse en el banco de datos.

Con tal fin se ha diseñado un sistema de clasificación y codificación de las denominaciones de los alimentos y sus componentes, basado en la clasificación del código alimentario español (15), el sistema EUROCODE (16) de EUROFOODS, y la consulta de bibliografia específica (17-19). En dicho sistema, a cada alimento se le asigna un código significativo y unívoco, que se divide en dos partes.

La primera indica el nombre, común o científico, y la clasificación jerárquica, a cuatro niveles, del alimento dentro del grupo de alimentos afines. El nivel primero identifica el grupo de alimentos de que trata, por ejemplo: productos lácteos o bebidas alcohólicas. Estos, en el segundo nivel, se dividen en grandes subgrupos, que a su vez se subdividen, en el tercer nivel, en grupos específicos de alimentos, y dentro de cada subgrupo específico, se irán incluyendo los alimentos concretos que constituirán el cuarto nivel (fig. 1).

La segunda parte del código se utiliza para precisar más el alimento, incluyendo información sobre las características que pueden influir en su composición. Por el momento se han considerado cuatro cualificadores que dan información sobre tipos de elaboración, estado de maduración, zona de procedencia o denominación de origen y variedad o materia prima.

Aunque hay otros aspectos de los alimentos que pueden afectar a sus componentes, creemos, en un principio, que con estos cualificadores se puede identificar de manera satisfactoria el alimento. De todas formas el sistema queda abierto para poder introducirle cualquier otra propiedad que se crea conveniente.

Figura 1

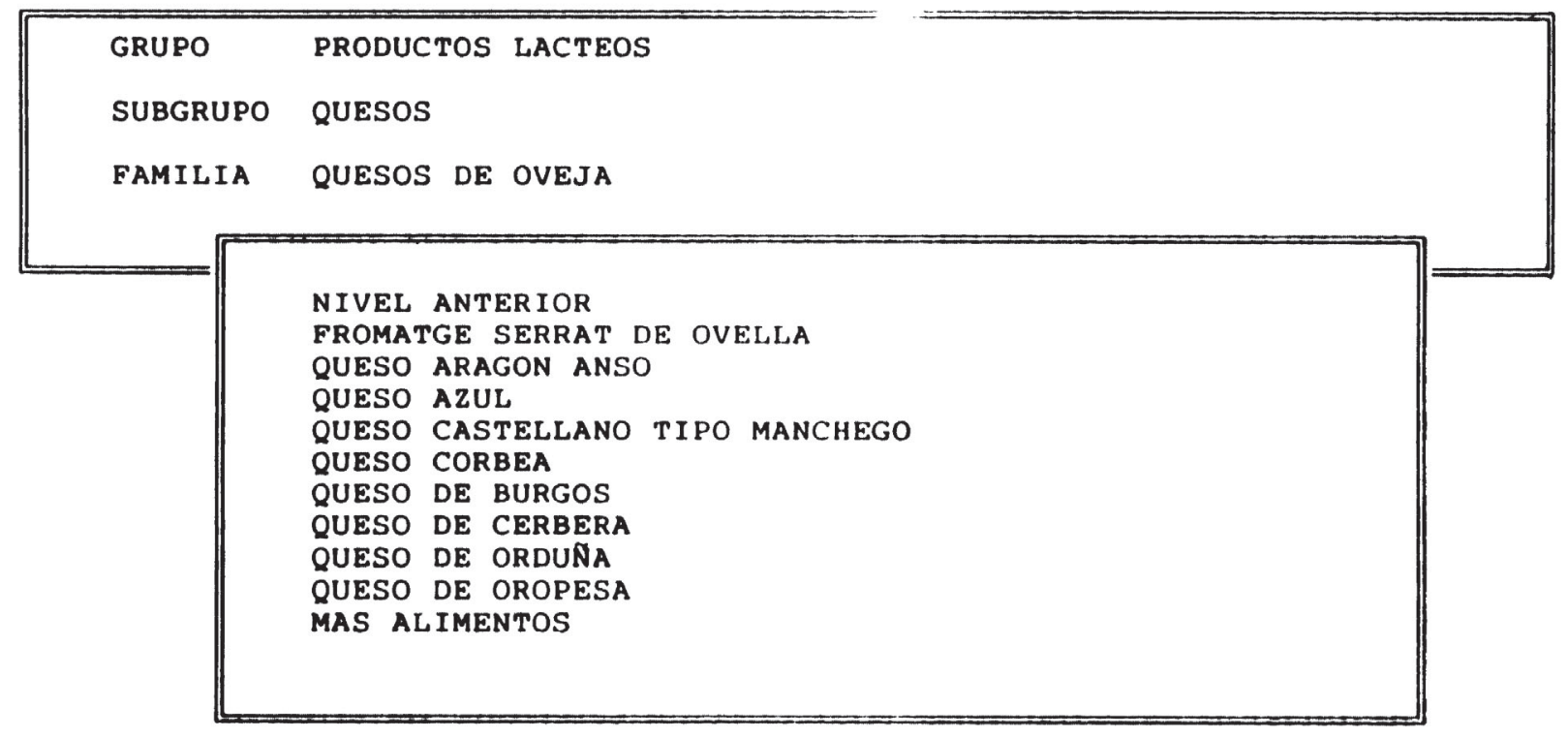




\subsection{Identificación de los componentes, su clasificación y medidas}

Al igual que pasa con los alimentos, los componentes deben identificarse sin ambigüedades. Esta identificación se hace mediante un sistema normalizado de nombres, sinónimos y códigos. Se han establecido 18 grandes grupos de componentes, en los que se agrupan 300 componentes químicos y características físicas, de acuerdo con las orientaciones de EUROFOODS (20-21). No quiere esto decir que para todos los alimentos tengan significado todos los componentes, ni que deban tener valores cada uno de ellos. Solamente se considerarán para cada alimento aquellos componentes que sean posibles y de los cuales se tengan datos. Los valores analíticos se pueden expresar en una de las tres unidades de medida que, para cada uno de los componentes, el sistema da opción.

\subsection{Identificación de las fuentes de información}

Los datos analíticos que se introducen en el sistema, y que afectan a un componente de un alimento, provendrán de muy distintas fuentes, con diferentes métodos y procedimientos. Es por esto necesario que dichas fuentes estén perfectamente identificadas, para dar la posibilidad de comprobar la información vertida en el banco de datos. Para ello, además de los datos referentes a los alimentos y sus componentes, también se incluirán datos referentes al documento del cual se ha obtenido la información. Con ello se consigue para cada dato no sólo que esté perfectamente determinada su clasificación y jerarquía, sino también su procedencia. Hay que considerar que, aunque el tratamiento automático sea eficiente, esto no garantiza la bondad de los resultados, ya que ésta depende de la calidad de los datos primarios.

\section{Estructura del banco de datos}

El banco de datos sobre componentes de alimentos españoles, desarrollado en el ICYT, se ha diseñado siguiendo el modelo relacional y se ha realizado en un PC/AT compatible utilizando el sistema de gestión de base de datos DBIII y su lenguaje de programación, para elaborar las funciones requeridas.

A partir de un menú principal se tiene acceso a otros dos menús que desarrollan las funciones de:

1) Actualización del banco de datos.

2) Recuperación de información.

Cada uno de ellos se va subdividiendo sucesivamente en módulos con funciones más específicas. Tanto en uno como en otro módulo el funcionamiento se ha establecido en base a menús que guían a los usuarios, así como un sistema de ayudas que se puede consultar en cualquier momento.

A continuación comentaremos someramente cada uno de estos módulos. 


\subsection{Actualización del banco}

Este módulo permite la actualización del bancoo de dantos, mediante la introducción de nueva información o la modificación de la existiente. La actualización se hace mediante tres módulos cuyas funciones es pecilicias \$wn: altas, bajas y modificaciones, ya sea de componentes, de alimentos, o dela identificación del documento, para lo que se han generado una serie de menús y ayudas.

\subsubsection{Módulo de altas}

El módulo de altas contempla tanto las altas de alimentos como las de componentes y las de identificación del doc umentio ctel cual se extraen los datos. Por eso, la introducción de información siempre acomenzzará con la identificación de la fuente, generando una referencia bibliográfic.a $\left(\boldsymbol{\Gamma}_{1}\right.$ g. 2). En ella se especifica el nombre y el tipo del documento, los autores, los o garnismos de trabajo de los mismos, o el organismo responsable de los datos, el nom lbre de la publicación que lo contiene, si es periódica o si no lo es, el nom bre delecitor de la publicación, el ISSN/ISBN, año, volumen, número, páginas. De esta:s caumpos son obligatorios el título, tipo y año de publicación del documento, assiccomoo el organismo; los demás son opcionales.

A cada referencia bibliográfica el sistema le asi gna unı identificador que será el campo de unión de la referencia bibliográfica con tod os 1 .os datos pertenecientes a ella.

Creada la referencia, se especifica el alimento det cual se quieren introducir datos analíticos. Solamente es obligatorio in tro ducir el mombre del alimento, y si se conoce o se desea, éste puede cualificarse más introcluciendo la materia prima, el método de elaboración, el estado de envejecimient $\infty$ o maduración, y la zona

\section{Figura 2}

ALTA A UNA REFERENCIA CORMLLIETh

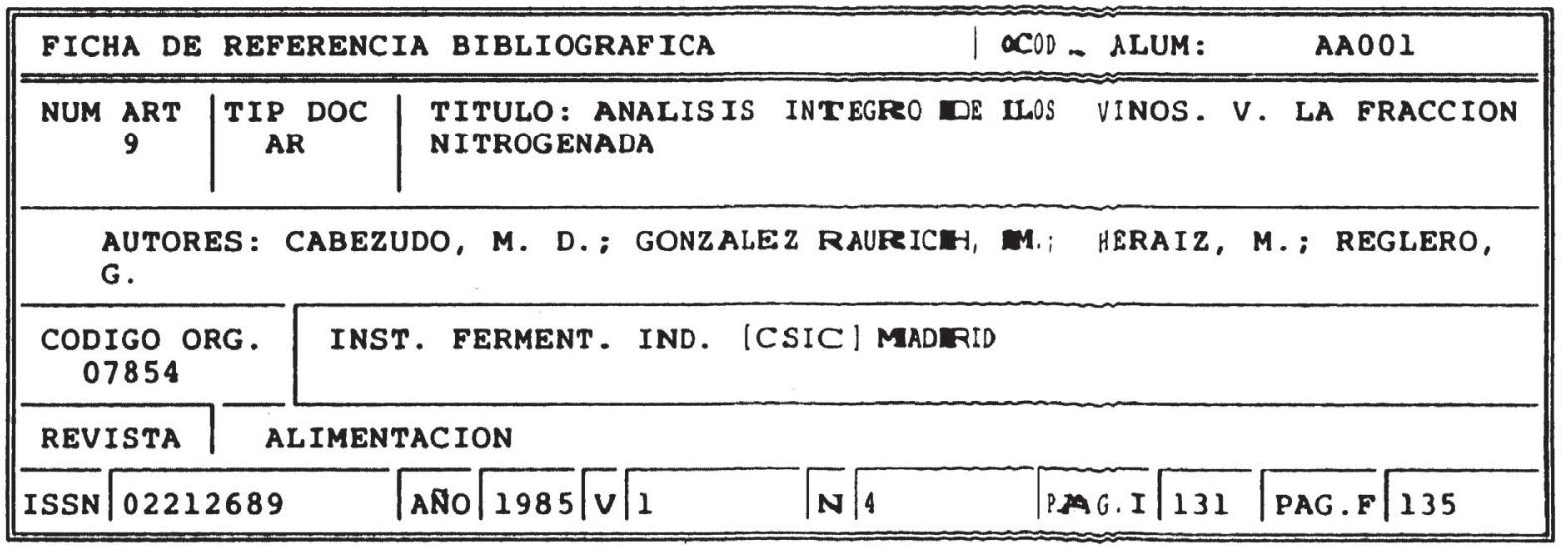

No DE REFERENCIA ASIGNADo. Pulse teclapiara continuar... 
geográfica o denominación de origen. Cuantas más especificaciones se hagan en la introducción de los datos, más concreta puede ser la recuperación de la información.

Una vez que el alimento ha sido aceptado por el sistema, éste pide que se especifique el componente, determinando primero el grupo al que pertenece, el método analítico, y las unidades de medida en las que se van a expresar los valores. Se da la opción de elegir entre tres unidades previamente establecidas. En los valores pueden especificarse la media, la máxima y la mínima, la desviación típica, y el número de muestras que han originado esos valores (fig. 3).

Figura 3

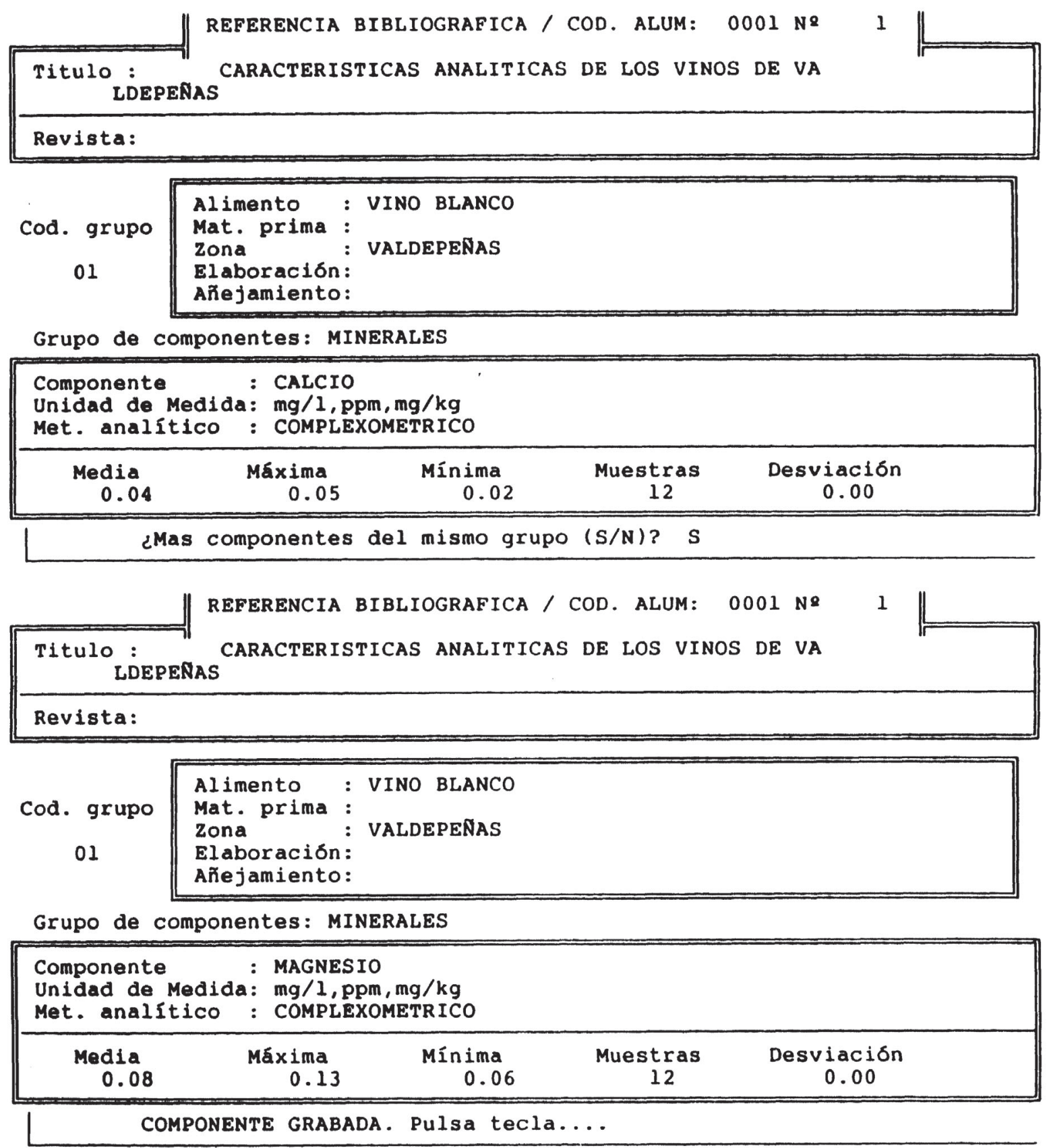




\subsubsection{Módulo de modificaciones}

Este módulo va a permitir, mediante una serie de menús, modificar cualquier dato introducido, a nivel de componente, de alimento, o de referencia bibliográfica.

Previamente a cualquier modificación que se quiera hacer, se debe especificar correctamente la identificación de los datos. Es decir, si queremos modificar algún valor analítico, se debe determinar el componente y alimento a los cuales corresponda, especificando todos los cualificadores de igual manera que se hizo en la toma de datos. Además tendrá que especificarse el identificador de la referencia bibliográfica de la que se han sacado los datos. Si esto no se hace correctamente, el sistema no permite la modificación.

\subsubsection{Módulo de bajas}

Al igual que en el caso de las modificaciones, el sistema permite dar de baja cualquier dato siempre que su identificación sea correcta e igual a como se hizo en la toma de datos (fig. 4). Esta máxima especificación para la verificación de los datos tiene su fundamento en la dependencia de cada nivel de información del nivel anterior. La baja de información en un determinado nivel supone la consiguiente eliminación de los datos dependientes de él en niveles inferiores. Así, la eliminación de un determinado alimento lleva consigo la eliminación de todos sus componentes, y la eliminación de una referencia bibliográfica supone eliminar del banco todos los datos relativos a ella, ya sean alimentos o componentes.

Figura 4

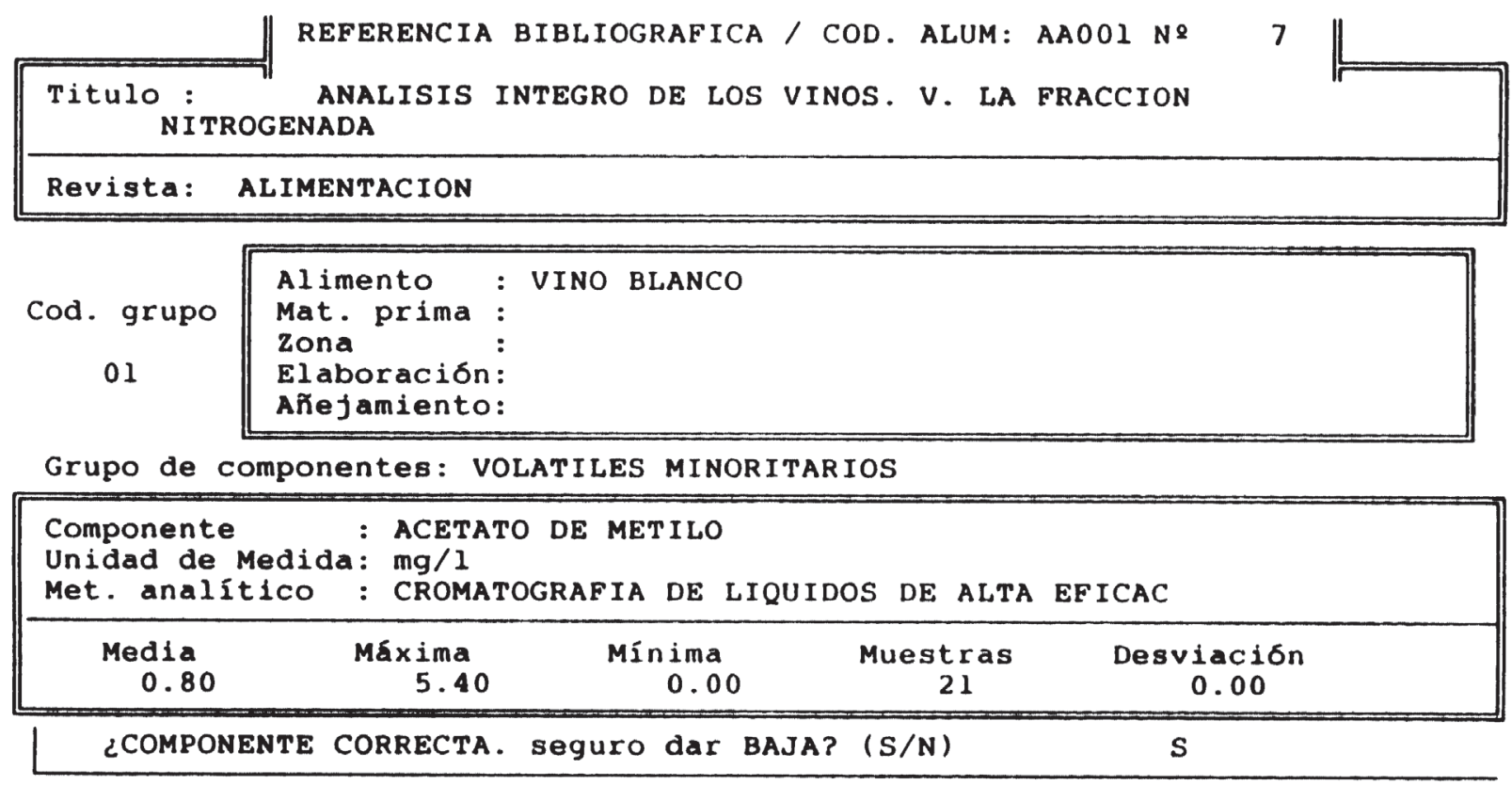




\subsection{Recuperación de información}

La información del banco de datos puede consultarse y recuperarse a través de las distintas opciones ofrecidas por este módulo (fig. 5).

Comentaremos a continuación estas opciones.

\section{Figura 5}

MENU DE RECUPERACIONES

\begin{tabular}{l} 
A. RECUPERACION DE ALIMENTOS POR SU COMPOSICION \\
B. RECUPERACION DE COMPONENTES DE UN ALIMENTO \\
C. RECUPERACION DE AUTORES \\
D. RECUPERACION DE REFERENCIAS \\
E. CLASIFICACION DE ALIMENTOS \\
\hline SALIR
\end{tabular}

\section{a) Recuperación de alimentos por su composición}

Esta opción permite la recuperación de alimentos atendiendo al contenido de determinados componentes o a la exclusión de otros. Para su funcionamiento, el sistema va pidiendo los componentes que debe contener el alimento y los que deben faltar. Una vez especificados éstos, el sistema proporciona el número de alimentos que lo cumplen y la visualización de cada uno de ellos con sus cualificadores (fig. 6).

\section{Figura 6}

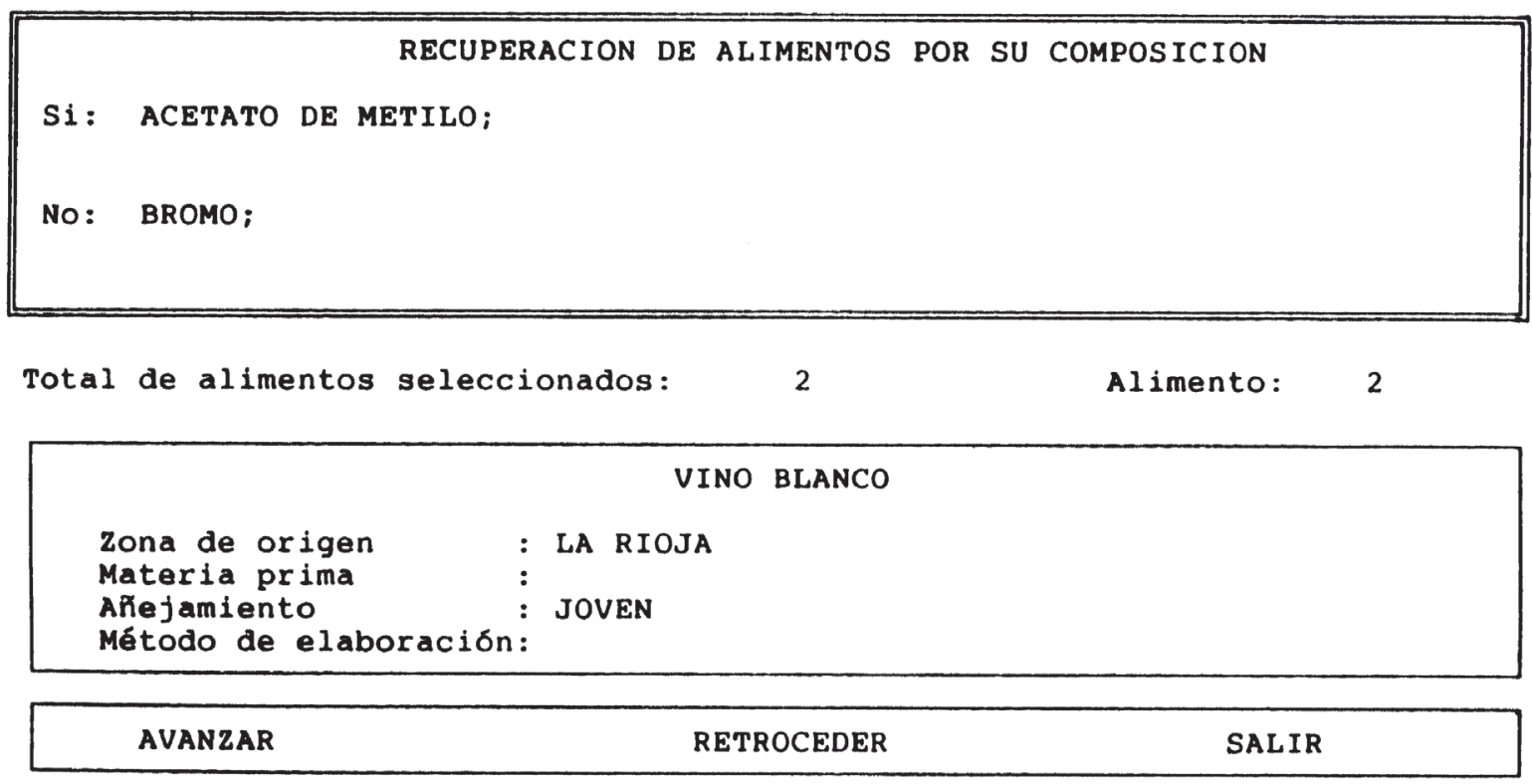




\section{b) Recuperación de los componentes de un alimento}

Con esta opción se recuperan los valores analíticos de los componentes de un alimento. Se puede optar por obtener todos los componentes de los cuales haya valores, o solamente los valores de determinados componentes que se especifican. Para ello hay que precisar el alimento del cual se quieren los datos. El sistema también permite diferentes grados de profundidad en la identificación del alimento, con lo que la recuperación será más o menos concreta. Es decir, se puede preguntar por un alimento dando su nombre solamente o definiéndolo más añadiendo uno o varios de sus cualificadores, tales como métodos de elaboración, madurez, materia prima o zona geográfica.

El resultado de la consulta proporciona, además de los valores máximo, mínimo, media y desviación típica, las unidades de medida, número de muestras y método analítico que ha dado origen a esos resultados, y el identificativo de la referencia de donde se han extraido (fig. 7).

\section{Figura 7}

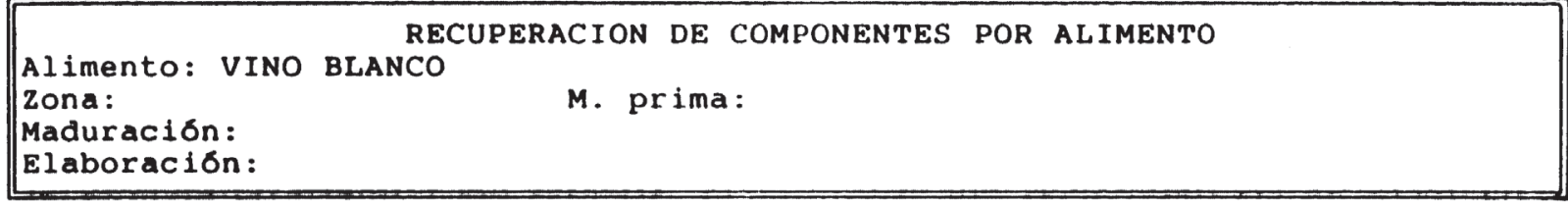

\begin{tabular}{|c|c|c|c|c|c|c|c|c|}
\hline COMPONENTE & |MET| & $|\mathbf{u}|$ & MEDIA & $\mid$ MAXIMA| & MIN IMA & DESV I & | MUES | & | REFERENC \\
\hline $\begin{array}{l}\text { MAGNESIO } \\
\text { POTASIO } \\
\text { CALCIO } \\
\text { HIERRO } \\
\text { 3-METIL-1-BUTANOL } \\
\text { 2-METIL-1-BUTANOL } \\
\text { ACETATO DE METILO }\end{array}$ & $\left|\begin{array}{lll}0 & 2 & 0 \\
0 & 1 & 3 \\
0 & 2 & 0 \\
0 & 1 & 3 \\
0 & 0 & 6 \\
0 & 0 & 6 \\
0 & 0 & 6\end{array}\right|$ & $\left|\begin{array}{l}\mathbf{A} \\
\mathbf{A} \\
\mathbf{A} \\
\mathbf{A} \\
\mathbf{A} \\
\mathbf{A} \\
\mathbf{A}\end{array}\right|$ & $\begin{array}{r}0.08 \\
1.00 \\
0.04 \\
0.01 \\
206.20 \\
53.90 \\
0.80\end{array}$ & $\left|\begin{array}{r}0.13 \\
1.21 \\
0.05 \\
0.01 \\
294.60 \\
79.00 \\
5.40\end{array}\right|$ & $\begin{array}{r}0.06 \\
0.92 \\
0.02 \\
0.00 \\
97.80 \\
22.50 \\
0.00\end{array}$ & $\begin{array}{l}0.00 \\
0.00 \\
0.00 \\
0.00 \\
0.00 \\
0.00 \\
0.00\end{array}$ & $\begin{array}{ll}1 & 2 \\
1 & 2 \\
1 & 2 \\
1 & 2 \\
2 & 1 \\
21 & 1 \\
2 & 1\end{array}$ & 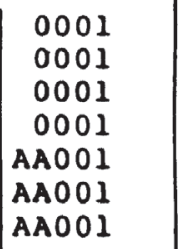 \\
\hline
\end{tabular}

SALIR $\rightarrow$ > ESC> \| AVANZAR - > PGdn> \| RETROCEDER - > PGUP>

\section{c) Recuperación de autores}

Esta opción permite recuperar los autores que han trabajado en un determinado alimento. Para ello se debe especificar el alimento deseado como se ha comentado anteriormente; puede acompañarse de todos aquellos cualificadores que precise. Cuantos más cualificadores se especifiquen, más concreto será el campo de trabajo de los autores que se recuperen. El resultado de la consulta dará todos los autores que han trabajado en el tema, especificando su nombre, su lugar de trabajo y el número total de referencias de cada autor en dicho alimento (fig. 8). 
Figura 8

Alimento: VINO BLANCO

Zona:

Maduración:

Elaboración:

RECUPERACION DE AUTORES POR ALIMENTO

\begin{tabular}{|c|c|c|}
\hline AUTORES & COD.ORG & TOT. REF \\
\hline $\begin{array}{l}\text { GARCIA, J. } \\
\text { LOPEZ, R. } \\
\text { ARAGON I. } \\
\text { MORENO GARCIA, J. } \\
\text { QUIRALTE CRESPO, J. } \\
\text { CABEZUDO, M. D. } \\
\text { CACERES, I. } \\
\text { GONZALEZ RAURICH, M. }\end{array}$ & $\begin{array}{l}01155 \\
01155 \\
02283 \\
02305 \\
02305 \\
07854 \\
07854 \\
07854\end{array}$ & $\begin{array}{l}1 \\
1 \\
1 \\
1 \\
1 \\
3 \\
1 \\
2\end{array}$ \\
\hline
\end{tabular}

SALIR $\rightarrow\langle$ ESC $>||$ AVANZAR $\rightarrow\langle$ Pgdn $>||$ RETROCEDER $\rightarrow\langle$ Pgup $>||$

\section{d) Recuperación de referencias}

Al igual que en la opción anterior, partiendo de la especificación del alimento se recuperarán las referencias que tratan de él. Además del número de referencias, éstas pueden visualizarse individualizadamente.

\section{e) Clasificación de alimentos}

Mediante esta opción el sistema permite, especificando un alimento sin cualificadores, conocer su clasificación jerárquica dentro del grupo de alimentos afines (véase fig. 1) que se ha considerado en el banco de datos. Mediante los cursores, se puede recorrer la estructuración de los alimentos y visualizar el contenido de los niveles anteriores y posteriores del alimento o grupo de alimentos que se está considerando.

\section{Bibliografia}

1. McCANCE, R. A.; WIDDOWSON, E. M. The Chemical Composition of Foods, Medical Research Council Spec. Rpt. Ser., 235, Londres, 1940.

2. HEINTZE, D.; KLENSI, J.; RAND, W. M. International Directory of Food Composition Tables, International Network of Food Data System, 1988.

3. ANDUJAR ARIAS, M. M.; MOREIRAS-VARELA, O.; GIL EXTREMERA, F. Tablas de composición de alimentos, Instituto de Nutrición (CSIC), 1987.

4. HERTZLER, A. A.; HOOVER, L. W. Development of food tables and use with computers, Journal of the American Dietetic Association, 70 (1), 20-31, 1977.

5. HEPBURN, F. N.; PERLOFF, B. P. The Nutrient Data Bank, 11 Cereal foods world, 24 (6), 224-225, 1979. 
6. SARTHOU, P. P. Infovin, un modele de Banque de Données sur le vin, RFOE, 102, 1986.

7. FAVIER, J. C.; FEINBERG, M. La Banque de données sur la composition des aliments: Un atour pour l'industrie laitière, Industrie Alimentaire Agricole. 103 (5), 404$410,1986$.

8. PETERSEN, B. J.; DOUGlaS, J. S.; FLEMING, K. H.; BUTRUM, R. The International Food Consumption/Composition Data-Base: FOODBASE, Third International Conference on Food Science and Technology. Information. Budapest, octubre 1989.

9. RAND, W. M.; YOUNG, V. R. Report of a planning conference concerning an international network of food data systems (INFOODS). The American Journal of Clinical Nutrition, 144-151, febrero 1984.

10. BUTRUM, R.; YOUNG, V. R. Development of Nutrient Data System for International Use: INFOODS (International Network of Food Data Systems), JNCI, 73 (6), diciembre 1984.

11. RAND, W. M.; KLENSIN, J. C. INFOODS: A world-wide effort to organize data on the composition of foods, Rev. Assoc. Canad. Diét, 49 (3), 158-163, 1988.

12. WEST, C. E.; ARAB, L. EUROFOODS: Working towards improvement and dissemination of food composition data in Europe, Food sciences and nutrition, 42F, 45-61, 1988.

13. WEST, C. E.; CASTENMILLER, J. J. M. Improvement of the Quality and Compatibility of Food Consumption and Food Composition Data in Europe, Report of the FLAIR workshop, Wageningen, julio 1990.

14. FERNANDEZ MUÑOZ, M. T.; GALBAN, C.; MENDEZ, A.; RIBAS, M. Creación de un banco de datos sobre características definitorias de los alimentos españoles: Productos lácteos, vinos y uvas, Proyecto de investigación n. ${ }^{\circ}$ PR8-0237-C07-07 CICYT, Madrid, abril 1989.

15. BARROS, C.; BAHLSEN, C.; PISA, J. Legislación Alimentaria de aplicación en España. (Clasificada por Alimentos), Sid-Alimentaria. 1989.

16. WITTLER, M.; ARAB, L. EUROFOODS, Proceedings of the second workshop. EUROCODE, Food Science and Nutrition, 42F, 1-7, 1988.

17. RIZEK, R. L.; PERLOFF, B. P.; POSATI, L. P. USDA's nutrient data bank, Food technology in Australia. 33 (3), 112-114, 1981.

18. BUTRUM, R.; PENNINGTON, J. Technology Systems Used for Food Composition Data Bases: An Historical Perspective, Computer Handliny and Dissemination of Data. PS Glaeser (Ed.) Elsevier Science Publishers BV (North Holland), CODAT A, 1987.

19. BUTRUM, R. Considerations in Designing a System for Description of Food and Flexible Retrieval of Relevant Data, The Role of Data in Scientific Progress, PS Glaeser (Ed.) Elsevier Science Publishers BV (North Holland), CODATA, 1985.

20. EUROFOODS. Working towards improvement and dissemination of food composition data in Europe. Appendix III: INFOODS recommended names in food composition tables, 1986.

21. KLENSIN, J. C.; FESKANICH, D.; LIN, V. Identification of food components for INFOODS data interchange. INFOODS/IS N40, 1-94, 1988. 